\title{
SUBCONTRA-CONTINUOUS FUNCTIONS
}

\author{
C.W. BAKER
}

Department of Mathematics Indiana University Southeast New Albany, Indiana 47150

(Received December 27, 1996)

\begin{abstract}
A weak form of contra-continuity, called subcontra-continuity, is introduced. It is shown that subcontra-continuity is strictly weaker than contra-continuity and stronger than both subweak continuity and sub-LC-continuity. Subcontra-continuity is used to improve several results in the literature concerning compact spaces.
\end{abstract}

KEY WORDS AND PHRASES: subcontra-continuity, contra-continuity, subweak continuity, subLC-continuity.

1991 AMS SUBJECT CLASSIFICATION CODE: 54C10

\section{INTRODUCTION}

In [1] Dontchev introduced the notion of a contra-continuous function. In this note we develop a weak form of contra-continuity, which we call subcontra-continuity. We show that subcontracontinuity implies both subweak continuity and sub-LC-continuity. We also establish some of the properties of subcontra-continuous functions. In particular it is shown that the graph of a subcontracontinuous function into a $T_{1}$-space is closed. Finally, we show that many of the applications of contra-continuous functions to compact spaces established by Dontchev [1] hold for subcontracontinuous functions. For example, we establish that the subcontra-continuous, nearly continuous image of an almost compact space is compact and that the subcontra-continuous, $\beta$-continuous image of an S-closed space is compact.

\section{PRELIMINARIES}

The symbols $X$ and $Y$ denote topological spaces with no separation axioms assumed unless explicitly stated. The closure and interior of a subset $A$ of a space $X$ are signified by $C l(A)$ and Int $(A)$, respectively. $\mathrm{A}$ set $A$ is regular open (semi-open, nearly open) provided that $A=\operatorname{Int}(C l(A))(A \subseteq C l(\operatorname{Int}(A)), A \subseteq \operatorname{Int}(C l(A)))$ and $A$ is regular closed (semi-closed) if its complement is regular open (semi-open). A set $A$ is locally closed provided that $A=U \cap F$, where $U$ is an open set and $F$ is a closed set.

DEFINITION 1. Dontchev [1]. A function $f: X \rightarrow Y$ is said to contra-continuous provided that for every open set $V$ in $Y, f^{-1}(V)$ is closed in $X$.

DEFINITION 2. Rose [2]. A function $f: X \rightarrow Y$ is said to be subweakly continuous if there is an open base $\mathcal{B}$ for the topology on $Y$ such that $C l\left(f^{-1}(V)\right) \subseteq f^{-1}(C l(V))$ for every $V \in \mathcal{B}$. 
DEFINITION 3. Ganster and Reilly [3]. A function $f: X \rightarrow Y$ is said to be sub-LCcontinuous provided there is an open base $\mathcal{B}$ for the topology on $Y$ such that $f^{-1}(V)$ is locally closed for every $V \in \mathcal{B}$.

DEFINITION 4. A function $f: X \rightarrow Y$ is said to be semi-continuous (Levine [4]) (nearly continuous (Ptak [5]), $\beta$-continuous (Abd El-Monsef et al. [6])) if for every open set $V$ in Y, $f^{-1}(V) \subseteq C l\left(\operatorname{Int}\left(f^{-1}(V)\right)\right)\left(f^{-1}(V) \subseteq \operatorname{Int}\left(C l\left(f^{-1}(V)\right)\right), f^{-1}(V) \subseteq C l\left(\operatorname{Int}\left(C l\left(f^{-1}(V)\right)\right)\right)\right)$.

DEFINITION 5. Gentry and Hoyle [7]. A function $f: X \rightarrow Y$ is said to be c-continuous if, for every $x \in X$ and every open set $V$ in $Y$ containing $f(x)$ and with compact complement, there exists an open set $U$ in $X$ containing $x$ such that $f(U) \subseteq V$.

\section{SUBCONTRA-CONTINUOUS FUNCTIONS}

We define a function $f: X \rightarrow Y$ to be subcontra-continuous provided there exists an open base $\mathcal{B}$ for the topology on $Y$ such that $f^{-1}(V)$ is closed in $X$ for every $\mathrm{V} \in \mathcal{B}$. Obviously contracontinuity implies subcontra-continuity. The following example shows that the reverse implication does not hold.

EXAMPLE 1. Let $X$ be a nondiscrete $T_{1}$-space and let $Y$ be the set $X$ with the discrete topology. Finally let $f: X \rightarrow Y$ be the identity mapping. If $\mathcal{B}$ is the collection of all singleton subsets of $Y$, then $\mathcal{B}$ is an open base for the topology on $Y$. Since $X$ is $T_{1}, f$ is subcontra-continuous with respect to $\mathcal{B}$. Obviously $f$ is not contra-continuous.

Subcontra-continuity is independent of continuity. The function in Example 1 is subcontracontinuous but not continuous. The next example shows that continuity does not imply subcontracontinuity.

EXAMPLE 2 Let $X=\{a, b\}$ be the Sierpinski space with the topology $\mathcal{T}=\{X, \emptyset,\{a\}\}$ and let $f: X \rightarrow X$ be the identity mapping. Obviously $f$ is continuous. However, any open base for the topology on $X$ must contain $\{a\}$ and $f^{-1}(\{a\})$ is not closed. It follows that $f$ is not subcontracontinuous.

Since closed sets are locally closed, subcontra-continuity implies sub-LC-continuity. We see from the following theorem that subcontra-continuity also implies subweak continuity.

THEOREM 1. Every subcontra-continuous function is subweakly continuous.

PROOF. Assume $f: X \rightarrow Y$ is subcontra-continuous. Let $\mathcal{B}$ be an open base for the topology on $Y$ for which $f^{-1}(V)$ is closed in $X$ for every $V \in \mathcal{B}$. Then for $V \in \mathcal{B}$, $C l\left(f^{-1}(V)\right)=f^{-1}(V) \subseteq f^{-1}(C l(V))$ and hence $f$ is subweakly continuous.

Since a subweakly continuous function into a Hausdorff space has a closed graph (Baker [8]), a subcontra-continuous function into a Hausdorff space has a closed graph. However, the following stronger result holds for subcontra-continuous functions.

THEOREM 2. If $f: X \rightarrow Y$ is a subcontra-continuous function and $Y$ is $T_{1}$, then the graph of $f, G(f)$, is closed.

PROOF. Let $(x, y) \in X \times Y-G(f)$. Then $y \neq f(x)$. Let $\mathcal{B}$ be an open base for the topology on $Y$ for which $f^{-1}(V)$ is closed in $X$ for every $V \in \mathcal{B}$. Since $Y$ is $T_{1}$, there exists $V \in \mathcal{B}$ such that $y \in V$ and $f(x) \notin V$. Then we see that $(x, y) \in\left(X-f^{-1}(V)\right) \times V \subseteq X \times Y-G(f)$. It follows that $G(f)$ is closed.

COROLLARY 1. If $f: X \rightarrow Y$ is contra-continuous and $Y$ is $T_{1}$, then the graph of $f$ is closed.

Long and Hendrix [9] proved that the closed graph property implies c-continuity. Therefore we have the following corollary.

COROLLARY 2. If $f: X \rightarrow Y$ is subcontra-continuous and $Y$ is $T_{1}$, then $f$ is c-continuous. 
The next two results are also implied by the closed graph property (Fuller [10]).

COROLLARY 3. If $f: X \rightarrow Y$ is subcontra-continuous and $Y$ is $T_{1}$, then for every compact subset $C$ of $Y, f^{-1}(C)$ is closed in $X$.

COROLLARY 4. If $f: X \rightarrow Y$ is subcontra-continuous and $Y$ is $T_{1}$, then for every compact subset $C$ of $X, f(C)$ is closed.

For a function $f: X \rightarrow Y$, the graph function of $f$ is the function $g: X \rightarrow X \times Y$ given by $g(x)=(x, f(x))$. We shall see in the following example that the graph function of a subcontracontinuous function is not necessarily subcontra-continuous.

EXAMPLE 3. Let $X=\{a, b\}$ be the Sierpinski space with the topology $\mathcal{T}=\{X, \emptyset,\{a\}\}$ and let $f: X \rightarrow \mathrm{X}$ be given by $f(a)=b$ and $f(b)=a$. Obviously $f$ is subcontra-continuous, in fact contra-continuous. Let $\mathcal{B}$ be any open base for the product topology on $X \times Y$. Then there exists $V \in \mathcal{B}$ for which $(a, b) \in V \subseteq\{(a, a),(a, b)\}$. We see that $V=\{(a, a),(a, b)\}$ and that, if $g: X \rightarrow$ $X \times X$ is the graph function for $f$, then $g^{-1}(V)=\{a\}$ which is not closed. Thus the graph function of $f$ is not subcontra-continuous.

However, the following result does hold for the graph function.

THEOREM 3. The graph function of a subcontra-continuous function is sub-LC-continuous.

PROOF. Assume $f: X \rightarrow Y$ is subcontra-continuous and let $g: X \rightarrow X \times Y$ be the graph function of $f$. Let $\mathcal{B}$ be an open base for the topology on $Y$ for which $f^{-1}(V)$ is closed in $X$ for every $V \in \mathcal{B}$. Then $\{U \times V: U$ is open in $X, V \in \mathcal{B}\}$ is an open base for the product topology on $X \times Y$. Since $g^{-1}(U \times V)=U \cap f^{-1}(V)$, we see that $g$ is sub-LC-continuous.

The graph function of a subweakly continuous function is subweakly continuous (Baker [8]) and the graph function of a sub-LC-continuous function is sub-LC-continuous (Ganster and Reilly [3]). It follows that the graph function in Example 3 is subweakly continuous and sub-LC-continuous but not subcontra-continuous. Therefore subcontra-continuity is strictly stronger than sub-LC-continuity and subweak continuity.

THEOREM 4. If $Y$ is a $T_{1}$-space and $f: X \rightarrow Y$ is a subcontra-continuous injection, then $X$ is $T_{1}$

PROOF. Let $x_{1}$ and $x_{2}$ be distinct points in $X$. Let $\mathcal{B}$ be an open base for the topology on $Y$ for which $f^{-1}(V)$ is closed in $X$ for every $V \in \mathcal{B}$. Since $Y$ is $T_{1}$ and $f\left(x_{1}\right) \neq f\left(x_{2}\right)$, there exists $V \in \mathcal{B}$ such that $f\left(x_{1}\right) \notin V$ and $f\left(x_{2}\right) \in V$. Then $x_{1} \in X-f^{-1}(V)$ which is open and $x_{2} \notin X-f^{-1}(V)$.

THEOREM 5. Let $A \subseteq X$ and $f: X \rightarrow X$ be a subcontra-continuous function such that $f(X)=A$ and $\left.f\right|_{A}$ is the identity on $A$. Then, if $X$ is $T_{1}, A$ is closed in $X$.

PROOF. Suppose $A$ is not closed. Let $x \in C l(A)-A$. Let $\mathcal{B}$ be an open base for the topology on $Y$ for which $f^{-1}(V)$ is closed for every $V \in \mathcal{B}$. Since $x \notin A$, we have that $x \neq f(x)$. Since $X$ is $T_{1}$, there exists $V \in \mathcal{B}$ such that $x \in V$ and $f(x) \notin V$. Let $U$ be an open set containing $x$. Then $x \in U \cap V$ which is open. Since $x \in C l(A),(U \cap V) \cap A \neq \emptyset$. Let $y \in(U \cap V) \cap A$. Since $y \in A, f(y)=y \in V$. So $y \in f^{-1}(V)$. Thus $y \in U \cap f^{-1}(V)$ and hence $U \cap f^{-1}(V) \neq \emptyset$. We see that $x \in C l\left(f^{-1}(V)\right)=f^{-1}(V)$ which is a contradiction. Therefore $A$ is closed.

The next result follows easily for the definition.

THEOREM 6. If $f: X \rightarrow Y$ is subcontra-continuous, then for every open set $V$ in $Y, f^{-1}(V)$ is a union of closed sets in $X$.

Obviously every function with a $T_{1}$-domain satisfies the above condition. However, as we see in the following example, a function with a $T_{1}$-domain can fail to be subcontra-continuous. It follows that the converse of Theorem 6 does not hold.. 
EXAMPLE 4. Let $X=\mathbb{R}$ with the usual topology and let $f: X \rightarrow X$ be the identity mapping. Since $X$ isconnected, $f$ is not subcontra-continuous. However, since $X$ is $T_{1}, f$ has the property that the inverse image of every (open) set is a union of closed sets.

\section{APPLICATIONS TO COMPACT SPACES}

In [1] Dontchev establishes that the image of an almost compact space under a contracontinuous, nearly continuous mapping is compact and that the contra-continuous image of a strongly S-closed space is compact. In this section, we strengthen both of these results by replacing contracontinuity with subcontra-continuity. The proofs mostly follow Dontchev's.

DEFINITION 6. Dontchev [1]. A space $X$ is almost compact provided that every open cover of $X$ has a finite subfamily the closures of whose members cover $X$.

THEOREM 7. The image of an almost compact space under a subcontra-continuous, nearly continuous mapping is compact.

PROOF. Let $f: X \rightarrow Y$ be subcontra-continuous and nearly continuous and assume that $X$ is almost compact. Let $\mathcal{B}$ be an open base for the topology on $Y$ for which $f^{-1}(V)$ closed in $X$ for every $V \in \mathcal{B}$. Let $\mathcal{C}$ be an open cover of $f(X)$. For each $x \in X$, let $C_{x} \in \mathcal{C}$ such that $f(x) \in C_{x}$. Then let $V_{x} \in \mathcal{B}$ for which $f(x) \in V_{x} \subseteq C_{x}$. Now $f^{-1}\left(V_{x}\right)$ is closed and nearly open. It follows that $f^{-1}\left(V_{x}\right)$ is clopen and hence that $\left\{f^{-1}\left(V_{x}\right): x \in X\right\}$ is a clopen cover of $X$. Since $X$ is almost compact, there is a finite subfamily $\left\{f^{-1}\left(V_{x_{1}}\right): i=1, \ldots, n\right\}$ for which $X=\bigcup_{i=1}^{n} C l\left(f^{-1}\left(V_{x_{1}}\right)\right)=\bigcup_{i=1}^{n} f^{-1}\left(V_{x_{1}}\right) \subseteq$ $\bigcup_{i=1}^{n} f^{-1}\left(C_{x_{i}}\right)$. Thus we have that $f(X) \subseteq \bigcup_{i=1}^{n} C_{x_{i}}$ and therefore that $f(X)$ is compact.

DEFINITION 7. Dontchev [1]. A space $X$ is strongly S-closed provided that every closed cover of $X$ has a finite subcover.

THEOREM 8. The subcontra-continuous image of a strongly S-closed space is compact.

PROOF. Let $f: X \rightarrow Y$ be subcontra-continuous and assume that $X$ is strongly S-closed. Let $\mathcal{B}$ be an open base for the topology on $Y$ for which $f^{-1}(V)$ is closed in $X$ for every $V \in \mathcal{B}$. Let $\mathcal{C}$ be an open cover of $f(X)$. For each $x \in X$, let $C_{x} \in \mathcal{C}$ with $f(x) \in C_{x}$. Then let $V_{x} \in \mathcal{B}$ for which $f(x) \in V_{x} \subseteq C_{x}$. Since $\left\{f^{-1}\left(V_{x}\right): x \in X\right\}$ is a closed cover of $X$ and $X$ is strongly S-closed, there is a finite subcover $\left\{f^{-1}\left(V_{x_{1}}\right): i=1, \ldots, n\right\}$ of $X$. Then we see that $f(X)=f\left(\bigcup_{i=1}^{n} f^{-1}\left(V_{x_{1}}\right)\right)=$ $\bigcup_{i=1}^{n} f\left(f^{-1}\left(V_{x_{i}}\right)\right) \subseteq \bigcup_{i=1}^{n} V_{x_{1}} \subseteq \bigcup_{i=1}^{n} C_{x_{i}}$ and hence that $f(X)$ is compact.

In [1] Dontchev also shows that the contra-continuous, $\beta$-continuous image of an S-closed space is compact. We extend this result by replacing contra-continuity with subcontra-continuity. The proof parallels that of Dontchev's.

DEFINITION 8. Mukherjee and Basu [11]. A space $X$ is S-closed provided that every semiopen cover of $X$ has a finite subfamily the closures of whose members covers $X$.

From Herrmann [12] , a space $X$ is S-closed if and only if every regular closed cover of $X$ has a finite subcover.

THEOREM 9. The subcontra-continuous, $\beta$-continuous image of an S-closed space is compact.

PROOF. Assume that $f: X \rightarrow Y$ is subcontra-continuous and $\beta$-continuous and that $X$ is Sclosed. Let $\mathcal{B}$ be an open base for the topology on $Y$ for which $f^{-1}(V)$ is closed in $X$ for every $V \in \mathcal{B}$. Let $\mathcal{C}$ be an open cover of $f(X)$. Then for each $x \in X$ there exists $\mathrm{C}_{x} \in \mathcal{C}$ for which $f(x) \in C_{x}$. For each $x \in X$, let $\mathrm{V}_{x} \in \mathcal{B}$ such that $f(x) \in V_{x} \subseteq C_{x}$. Since $f$ is subcontra-continuous, $\left\{f^{-1}\left(V_{x}\right): x \in X\right\}$ is a closed cover of $X$. The $\beta$-continuity of $f$ implies that $f^{-1}\left(V_{x}\right) \subseteq C l\left(\operatorname{Int}\left(C l\left(f^{-1}\left(V_{x}\right)\right)\right)\right)$ and therefore we see that $f^{-1}\left(V_{x}\right)=C l\left(\operatorname{Int}\left(f^{-1}\left(V_{x}\right)\right)\right)$ or that 
$f^{-1}\left(V_{x}\right)$ is regular closed. Since $X$ is S-closed, the regular closed cover $\left\{f^{-1}\left(V_{x}\right): x \in X\right\}$ has a finite subcover $\left\{f^{-1}\left(V_{x_{1}}\right): i=1, \ldots, n\right\}$. Then we have $f(X)=f\left(\bigcup_{i=1}^{n} f^{-1}\left(V_{x_{1}}\right)\right) \subseteq \bigcup_{i=1}^{n} V_{x_{1}} \subseteq$ $\bigcup^{n} C_{x_{i}}$ and therefore $f(X)$ is compact. $i=1$

In the above proof we showed that, if $f: X \rightarrow Y$ is subcontra-continuous and $\beta$-continuous, then there exists an open base $\mathcal{B}$ for the topology on $Y$ such that for every $V \in \mathcal{B}, f^{-1}(V)$ is regular closed and hence semi-open. Since unions of semi-open sets are semi-open (Arya and Bhamini [13]), it follows that inverse images of open sets are semi-open. Therefore we have the following theorem which strengthens the corresponding result for contra-continuous functions established by Dontchev [1].

THEOREM 10. Every subcontra-continuous, $\beta$-continuous function is semi-continuous.

\section{REFERENCES}

1. Dontchev, J. Contra-continuous functions and strongly S-closed spaces, Internat. J. Math \& Math. Sci. 19 (1996), 303-310.

2. Rose, D. A. Weak continuity and almost continuity, Internat. J. Math. \& Math Sci. I (1984), 311-318.

3. Ganster, M. and Reilly, I. L. Locally closed sets and LC-continuous functions, Internat. J. Math. \& Math. Sci. 12 (1989), 417-424.

4. Levine, N. Semi-open sets and semi-continuity in topological spaces, Amer. Math. Monthly 70 (1963), 36-41.

5. Ptak, V. Completeness and open mapping theorem, Bull. Soc. Math. France 86 (1958), 41-74.

6. Abd El-Monsef, M. E., El-Deeb, S. N., and Mahmoud, R. A. $\beta$-open sets and $\beta$-continuous mappings, Bull. Fac. Sci.Assiut Univ 12 (1983), 77-90.

7. Gentry, K. R. and Hoyle, H. B. C-continuous functions, Yokohama Math. J 18 (1970), 71-76.

8. Baker, C. W. Properties of subweakly continuous functions, Yokohama Math. J 32 (1984), 39-43.

9. Long, P. E. and Hendrix, M. D. Properties of c-continuous functions, Yokohama. Math. J. 22.(1974), 117-123.

10. Fuller, R. V. Relations among continuous and various noncontinuous functions, Pacific $J$ Math. 25 (1968), 495-509.

11. Mukherjee, M. N. and Basu, C. K. On S-closed and s-closed spaces, Bull.Malaysian Math. Soc. (Second Series) 15 (1992),1-7.

12. Herrmann, R. A. RC-convergence, Proc. Amer. Math. Soc. 75 (1979), 311-317.

13. Arya S. P. and Bhamini M. P. Some weaker forms of semi-continuous functions, Gantita 33 (1982), 124-134. 


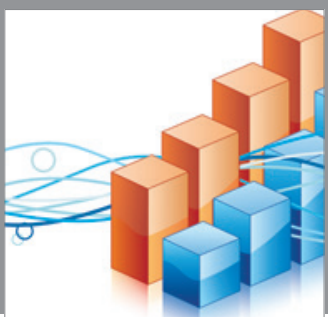

Advances in

Operations Research

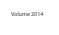

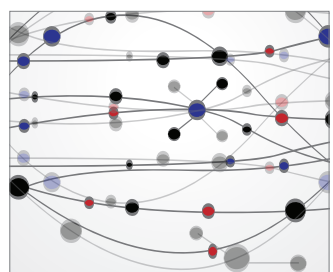

\section{The Scientific} World Journal
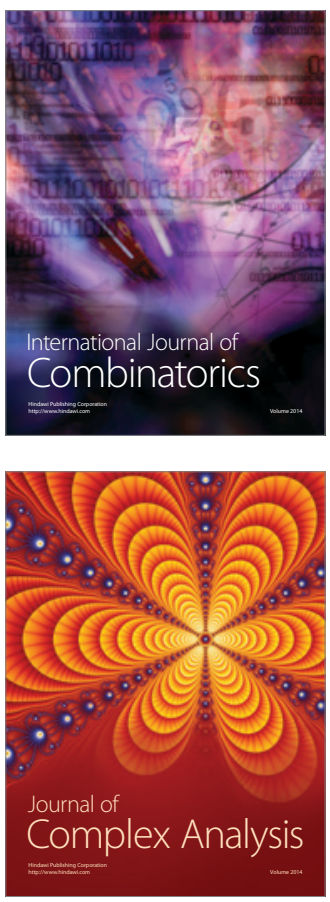

International Journal of

Mathematics and

Mathematical

Sciences
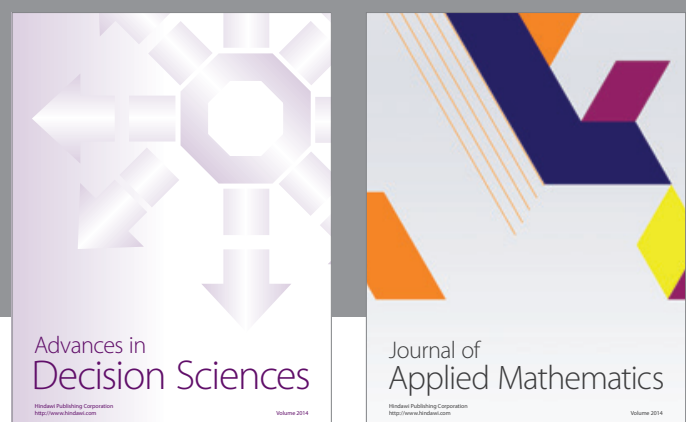

Journal of

Applied Mathematics
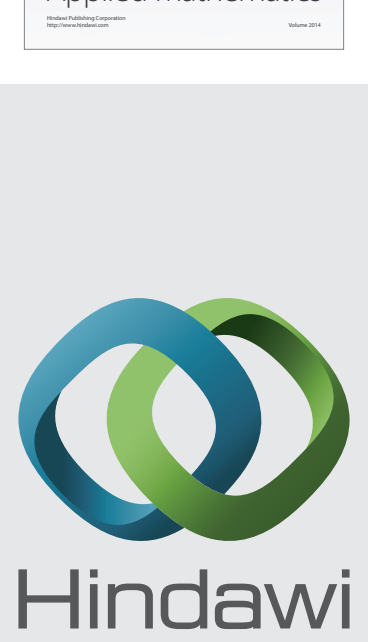

Submit your manuscripts at http://www.hindawi.com
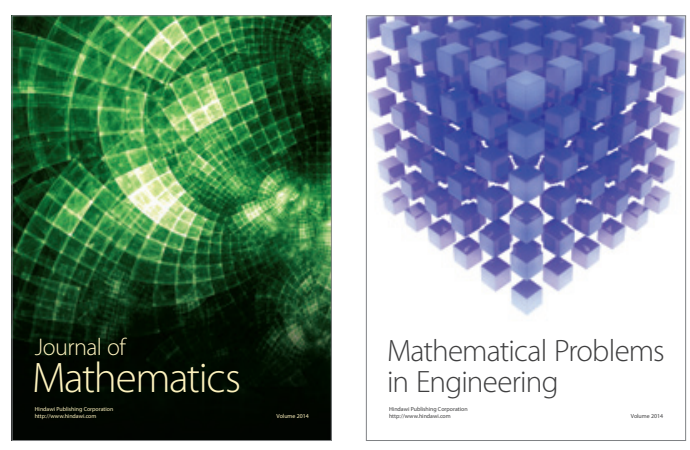

Mathematical Problems in Engineering
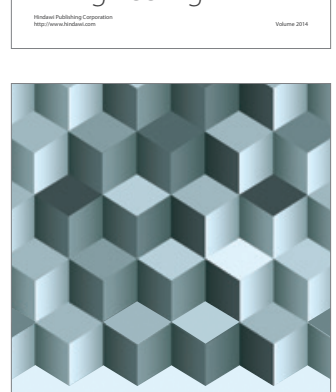

Journal of

Function Spaces
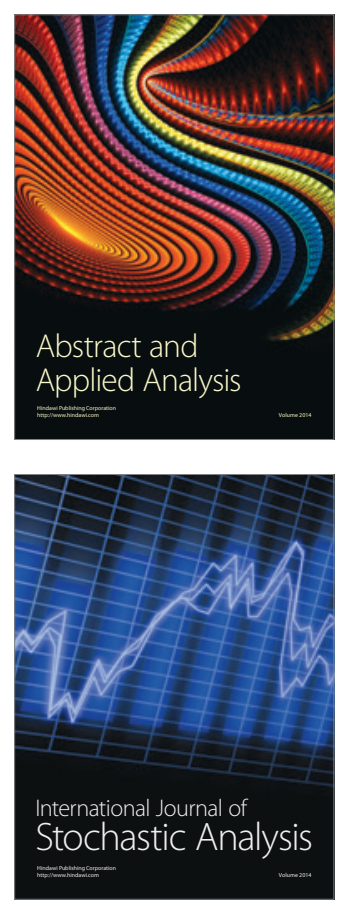

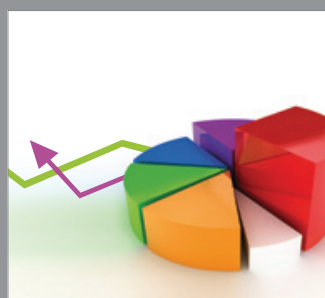

ournal of

Probability and Statistics

Promensencen
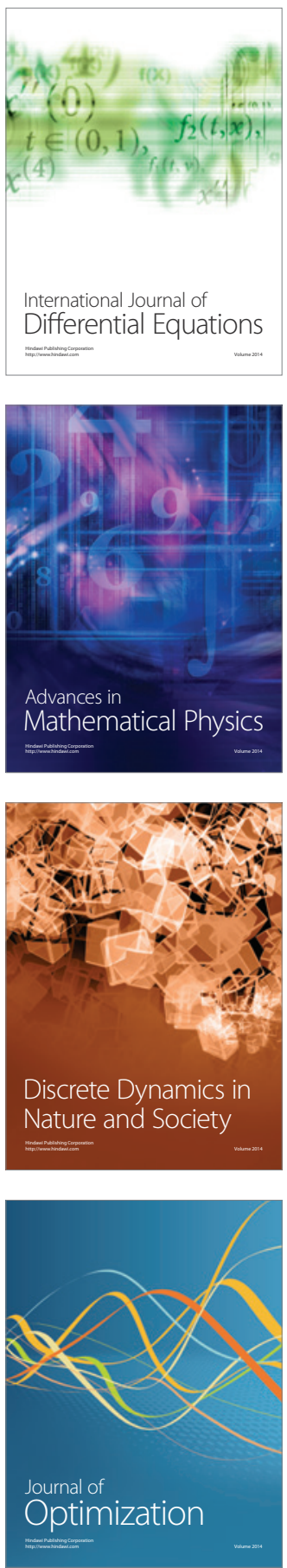